The Egyptian Journal of Hospital Medicine (October 2021) Vol. 85 (1), Page 2951-2955

\title{
Efficacy of Distal Intracoronary Epinephrine and Glycoprotein IIb/IIIa Inhibitors in the Treatment of No-reflow during Percutaneous Coronary Intervention; a Pilot Project.
}

\author{
${ }^{1}$ Mahmoud Tantawy, ${ }^{1}$ Ahmed Elbarbary, ${ }^{2}$ Ahmed Tamara, ${ }^{2}$ Ghada Selim \\ ${ }^{1}$ Cardiology Department, Faculty of Medicine, Misr University for Science and Technology (MUST), \\ ${ }^{2}$ Cardiology Department, Faculty of Medicine, Ain Shams University, Egypt \\ *Corresponding author: Mahmoud Tantawy, Mobile: (+20) 0121865587
}

\begin{abstract}
Background: No-reflow is considered a major percutaneous coronary intervention complication, especially in primary PCI. A variety of medications have been studied for no-reflow treatment, including intracoronary nitrates, verapamil, adenosine, glycoprotein IIb/IIIa inhibitors, and epinephrine. Glycoprotein IIb/IIIa inhibitors and epinephrine are the two most promising agents for the treatment of no-reflow.

Aim of work: evaluating epinephrine and glycoprotein IIb/IIIa inhibitors efficacy in treating no-reflow through local distal intracoronary injection in comparison to traditional intracoronary administration in the guiding catheter.

Subjects and methods: 30 patients undergoing PCI complicated by no-reflow phenomenon. Patients were randomized to either group I where they were treated by local distal intracoronary injection of GP IIb/IIIa inhibitor and epinephrine or to group II where they received the same medications via the traditional intracoronary injection. Primary outcomes were TIMI flow, corrected TIMI frame count and TMPG, and major adverse cardiac events within 48 hours was the secondary outcome. Results: Group I had significantly superior angiographic outcomes than group II. As regards TIMI II-III flow, $86.7 \%$ of patients achieved it in versus $53.3 \%$ in group II, CTFC was 8.2versus 9.9, and in group I, 80\% of patients achieved TMPG II-III, compared to $46.7 \%$ in group II. MACE was insignificantly different between both groups. Diabetes mellitus was found to be the only predictor to be negatively associated with TIMI flow.

Conclusion: Distal coronary artery local injection of a combination of GP IIb/IIIa inhibitors and epinephrine achieved superior angiographic outcomes in the treatment of refractory no-reflow in comparison with traditional intracoronary administration of the same medications.
\end{abstract}

Keywords: No-reflow, Intracoronary epinephrine, GP IIb/IIIa inhibitors.

\section{INTRODUCTION}

The No-reflow phenomenon is considered one of the nightmares in the catheter laboratory. It is known as inadequate myocardial perfusion without an apparent mechanical cause, like a coronary dissection of significant obstruction. Primary percutaneous coronary intervention (PCI) is more likely to result in no-reflow than elective PCI. Different mechanisms contribute to the no-reflow phenomenon, including distal microembolization, reperfusion injury, endothelial dysfunction, microvascular spasm, and myocardial edema ${ }^{(1)}$.

Multiple medications have been studied in the treatment of no-reflow. Some of them have shown benefits like nitrates ${ }^{(1,2)}$, calcium channel blockers ${ }^{(3)}$, adenosine ${ }^{(4)}$, glycoprotein IIb/IIIa inhibitors ${ }^{5}$, and epinephrine ${ }^{(6-8)}$. Most of these drugs were partially effective in the treatment of no-reflow but have shown variable results across different studies, and there is still a limited success rate in the treatment of refractory no-reflow ${ }^{(6)}$.

A meta-analysis ${ }^{(7)}$ has shown that intralesional injection of GP IIb/IIIa has better outcomes in preventing no-reflow than intracoronary (IC) injection as it gives better contact with the thrombus burden. A single study ${ }^{(3)}$ concluded that the usage of distal injection of epinephrine with or without verapamil had shown superior results than traditional IC injection of the same medications.

As the intracoronary (IC) usage of GP IIb/IIIa inhibitors and epinephrine are considered effective agents in the treatment of persistent no-reflow (8), local distal intracoronary injection of both medications may get more benefits.

AIM OF WORK: assessing epinephrine and glycoprotein IIb/IIIa inhibitors efficacy in treating no-reflow through local distal intracoronary injection in comparison to traditional intracoronary administration in the guiding catheter.

\section{SUBJECTS AND METHODS}

The present study included 30 documented coronary artery disease patients who underwent either rescue or primary PCI complicated by no-reflow.

\section{Ethical approval:}

MUST university Ethical Committee Board approved the study. Each patient enrolled in the study signed an informed consent form. This work has been carried out in accordance with The Code of Ethics of 
the World Medical Association (Declaration of Helsinki) for studies involving humans.

The study began from April 2018 to December 2019.

Two groups of patients were assigned randomly:

1) Group I received the bolus dose of GP IIb/IIIa inhibitor according to body weight followed by $100 \mathrm{mcg}$ of epinephrine through microcatheter or aspiration device or perfusion balloon at the target no-reflow coronary artery distal segment.

2) Group II received the same medications with the same doses through the traditional intracoronary route via a well-engaged guiding catheter.

Before and after injection of GP IIb/IIIa inhibitors and epinephrine, Myocardial Blush, Corrected TIMI Frame Count, and Thrombolysis In Myocardial Infarction (TIMI) flow were judged blindly by two independent operators for all cases.

Angiographic Coronary flow analysis ${ }^{9} ;$ According to the TIMI study criteria, the coronary flow was graded as follows:

- TIMI grade 0: total occlusion, with no contrast flow distal to the occlusion site associated with the infarct.

- TIMI grade I: Contrast penetrates around the obstruction site, but there is limited distal antegrade perfusion.

- TIMI grade II: Reduced rate of contrast entry and clearance into and out of the distal coronary artery bed.

- TIMI grade III: Contrast entry and clearance rates into and out of the distal coronary artery bed are normal.

\section{"Corrected TIMI Frame Count ${ }^{(10)}$ :}

Corrected TIMI frame count was also calculated in the culprit's vessel as a marker of the velocity of the coronary flow, as follows:

The first frame for counting was the frame in which the dye completely enters the artery, contacting both borders of the artery origin; with the dye antegrade motion.

The last frame is the one in which dye first enters the distal landmark branch.

The distal landmark branches used for counting were: left anterior descending coronary (LAD) distal bifurcation, the distal bifurcation of the segment with the longest total distance that includes the culprit lesion in the left circumflex coronary artery (LCX), and the first branch of the posterolateral artery in the right coronary artery (RCA)."

\section{$\underline{\text { Myocardial Blush }}^{(9)}$ :}

Myocardial blush was graded according to the TMPG system:
- Grade 0 TIMI Myocardial Perfusion: Dye is unable to enter the microvasculature. Either a little or full of ground glass appearance ("blush") absence, or opacification of the myocardium indicating a lack of tissue-level perfusion.

- Grade 1 TIMI Myocardial Perfusion: Dye diffuses slowly into the microvasculature but does not exit. In the distribution of the culprit lesion, there is a ground-glass appearance ("blush") of the myocardium and dye staining is visible on successive injections (approximately 30 seconds between injections).

- Grade 2 TIMI Myocardial Perfusion: Dye entry and exit from the microvasculature are delayed. Dye strongly persists after three cardiac cycles of the washout phase, and either does not or only minimally diminish in intensity during washout.

- Grade 3 TIMI Myocardial Perfusion: Normally, the dye enters and leaves the microvasculature. Dye is fade out or only mildly to moderately persistent after three cardiac cycles of the washout phase and significantly diminishes in intensity during the washout phase.

The RCA: The optimum view for the RCA was determined to be the left anterior oblique (LAO) view with cranial angulation. The LAD: the optimal view for assessing the LAD and circumflex is the left lateral projection. The Circumflex: Left lateral projection is the optimal view for circumflex and LAD assessment.

\section{Statistical Analysis}

SPSS (statistical package for the social sciences, version 12) were used to analyze the data.

Mean and standard deviation (SD) were used to describe quantitative variables, which were compared by unpaired t-test. Chi-square test was utilized for comparing qualitative variables which were presented as frequency and percentage. Multivariate logistic regression analysis: Odds ratios (OR) with 95\% confidence intervals were computed in to assess the overall association between each possible risk factor and its effect on TIMI flow. High significant $\mathrm{p}$-value $<0.01$. P-value $<0.05$ was considered significant.

\section{RESULTS}

This study included 30 patients with no-reflow who were randomly assigned into two groups, each with 15 patients. Group I received the treatment at the distal part of the target vessel, and group II received the same treatment intracoronary through the guiding catheter.

Demographic and basic data, including age, sex, risk factors, baseline echocardiography, target vessel, and type of intervention, were nearly similar in both groups (Tables $1,2)$. 
Table (1): Demographic data and risk factors in the studied groups:

\begin{tabular}{|l|l|l|l|}
\hline Variables & Group I (N=15) & Group II (N=15) & P \\
\hline Age & $58.8 \pm 13$ & $60 \pm 15$ & $>0.05$ \\
\hline Gender & & & \\
Male & $9(60 \%)$ & $8(53.3 \%)$ & $>0.05$ \\
Female & $6(40 \%)$ & $7(46.7 \%)$ & \\
\hline Diabetic & $5(33.3 \%)$ & $6(40 \%)$ & $>0.05$ \\
\hline Hypertensive & $11(73.3 \%)$ & $10(66.6 \%)$ & $>0.05$ \\
\hline Smoker & $6(40 \%)$ & $5(33.3 \%)$ & $>0.05$ \\
\hline Dyslipidemia & $3(20 \%)$ & $4(26.6 \%)$ & $>0.05$ \\
\hline Family history & $2(13.3 \%)$ & $4(26.6 \%)$ & $>0.05$ \\
\hline
\end{tabular}

Table 2: Echocardiographic and Angiographic data before drug injection:

\begin{tabular}{|c|c|c|c|}
\hline 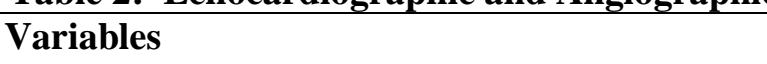 & Group I & Group II & $\mathbf{P}$ \\
\hline Ejection Fraction (EF\%) & $45.9 \pm 9$ & $47.2 \pm 13$ & $>0.05$ \\
\hline $\begin{array}{l}\text { Type of intervention: } \\
\text { - Primary } \\
\text { - Rescue }\end{array}$ & $\begin{array}{ll}\text { - } & 3(20 \%) \\
\text { - } & 12(80 \%) \\
\end{array}$ & $\begin{array}{ll}\text { - } & 4(26.7 \%) \\
\text { - } & 11(73.3 \%)\end{array}$ & $>0.05$ \\
\hline $\begin{array}{c}\text { Target vessel TIMI flow before drugs injection: } \\
\qquad \quad \text { TIMI } 0 \\
\bullet \quad \text { TIMI } 1\end{array}$ & $\begin{array}{ll}\cdot & 9(60 \%) \\
\text { - } & 6(40 \%)\end{array}$ & $\begin{array}{ll}- & 8(53.3 \%) \\
\text { - } & 7(46.7 \%)\end{array}$ & $>0.05$ \\
\hline RCA & $2(13.3 \%)$ & $1(6.7 \%)$ & \multirow{5}{*}{$>0.05$} \\
\hline LCX & $3(20 \%)$ & $2(13.3 \%)$ & \\
\hline LAD & $7(46.7 \%)$ & $8(53.3 \%)$ & \\
\hline $\mathbf{O M}$ & $2(13.3 \%)$ & $3(20 \%)$ & \\
\hline PDA & $1(6.7 \%)$ & $1(6.7 \%)$ & \\
\hline
\end{tabular}

RCA=right coronary artery, LCX= left circumflex coronary artery, LAD= left anterior descending coronary artery, $\mathrm{OM}=$ obtuse marginal, PDA =posterior descending coronary artery.

Results following drug injection show that group I had significantly superior angiographic outcomes than group II (Table 3). While 13 patients from group I has achieved TIMI II-II in comparison with only 8 patients in group II (86.7\% versus $53.3 \%, \mathrm{P}=0.043)$. Group I showed a significantly lower CTFC $(\mathrm{P}=0.041)$. Moreover, 12 patients in group I and 7 patients in group II achieved TMPG II-II ( $80 \%$ versus $46.7 \%, \mathrm{P}=0.048)$.

Table 3: Angiographic data after drug injection:

\begin{tabular}{|l|c|c|c|}
\hline Variable & Group I & Group II & P \\
\hline TIMI II-III & $13(86.7 \%)$ & $8(53.3 \%)$ & $0.043^{*}$ \\
\hline TMPG II-III & $12(80 \%)$ & $7(46.7 \%)$ & $0.048^{*}$ \\
\hline CTFC & $8.2 \pm 2.01$ & $9.9 \pm 2.32$ & $0.041^{*}$ \\
\hline \multicolumn{2}{|r|}{ "significant }
\end{tabular}

The studied groups showed no significant difference $(\mathrm{P}=0.07)$ as regards 48 hours major adverse cardiac events (MACE). (Table 4)

Table 4: Secondary outcome: 48 hours major adverse cardiac events (MACE):

\begin{tabular}{|l|c|c|c|}
\hline Variables & Group I (N=15) & Group II $(\mathrm{N}=15)$ & \multirow{2}{*}{ P } \\
\hline UA & 0 & 0 & \multirow{3}{*}{$>0.05 \mathrm{NS}$} \\
\hline TLR & 0 & 0 & \\
\hline MI & $1(6.7 \%)$ & 0 & \\
\hline Death & $1(6.7 \%)$ & $3(20 \%)$ & \\
\hline CABG & 0 & $1(6.7 \%)$ & \\
\hline Total MACE & $2(13.3 \%)$ & $4(26.6 \%)$ & \\
\hline
\end{tabular}

UA; unstable angina, TLR; target lesion revascularization, MI; myocardial infarction $M A C E$; major adverse cardiac events, CABG; coronary artery bypass graft. 
Table (5): Risk factors as predictors of TIMI flow by using multivariate regression analysis:

\begin{tabular}{|l|c|c|c|c|c|}
\hline \multirow{2}{*}{ Risk factors } & \multirow{2}{*}{ B } & \multirow{2}{*}{ OR } & \multicolumn{2}{|c|}{ 95\% C.I. } & \multirow{2}{*}{ p-value } \\
\cline { 4 - 5 } & & & Lower & Upper & \\
\hline Age >60 years & -0.148 & 0.564 & 0.228 & 2.370 & 0.231 \\
\hline Male & -0.451 & 0.875 & 0.365 & 4.034 & 0.123 \\
\hline Diabetic & -0.144 & 0.745 & 0.340 & 3.468 & $0.024^{*}$ \\
\hline Hypertensive & -0.131 & 0.910 & 0.798 & 5.145 & 0.319 \\
\hline Smoking & -0.163 & 0.616 & 0.446 & 2.921 & 0.201 \\
\hline Dyslipidemia & -0.050 & 0.937 & 0.279 & 5.811 & 0.139 \\
\hline Family history & -0.252 & 0.547 & 0.221 & 2.299 & 0.224 \\
\hline
\end{tabular}

B: Regression coefficient, Odds ratio, SE: Standard error, CI: Confidence interval, "significant

Diabetes mellitus was the only predictor identified to be negatively associated with TIMI flow in multivariate regression analysis, encompassing predictors such as age, gender, hypertension, smoking, and hypercholesterolemia and family history (FH) of coronary artery disease (table 5).

\section{DISCUSSION}

No reflow is the lack of myocardial perfusion despite the opening of epicardial coronaries that may occur in elective or primary PCI. It has been closely linked to the development of malignant arrhythmias, reduction in ejection fraction ${ }^{(11)}$, and elevated mortality risk and is therefore critically important to try to prevent it and treat it to preserve viable myocardium.

While a variety of mechanical and pharmacological strategies have been investigated to prevent no-reflow, there exists scanty literature on its treatment.

Epinephrine is one of the drugs used in addressing this phenomenon. It has a dual advantage as it is a potent Beta 2 agonist inducing vasodilatation, while its vasoconstricting effect through its alpha-agonist activity helps in antagonizing the hypotension resulting from noreflow ${ }^{(12)}$. Intracoronary injection of epinephrine has been suggested to be safe and effective in cases of noreflow (6-8). Similarly, IIb/IIIa platelet receptor antagonists have shown some promise ${ }^{(5,13)}$.

In this study, we tested the combination regimen of epinephrine and GpIIb/IIIa to augment the vasodilatation and antiplatelet benefit. In addition, this is the only pilot study that we are aware of that seeks to compare the delivery of both drugs through a guiding catheter to its delivery in the distal microcirculation, believed to avoid its dilution and allow better penetration in the microcirculation, thus greater bioavailability and higher efficacy. This is also one of fewer studies that focus on treating no-reflow rather than preventing it ${ }^{(5,13)}$.

Our study revealed that epinephrine and GpIIb/IIIa inhibitor local delivery resulted in significantly better angiographic outcomes and no-reflow treatment results compared to their in-catheter delivery. Thirteen patients $(86.7 \%)$ in the distal delivery arm achieved TIMI II-III flow compared to only $53.3 \%$ in group II. Furthermore, CTFC was 8.2+2.01 in group I versus 9.9+2.32. In group I, $80 \%$ of patients achieved TMPG II-III versus $46.7 \%$ in group II.
Aksu et al. ${ }^{(6)}$ showed positive results using local epinephrine delivery in primary PCI, which prompted other investigators to evaluate its use in treating noreflow. The intracoronary epinephrine efficacy was further demonstrated in 2021 by Eliano et $\boldsymbol{a l}$. ${ }^{(7)}$ who compared intracoronary epinephrine to conventional treatments alone (including adenosine, Glycoprotein $\mathrm{IIb} / \mathrm{III}$ inhibitors, IC nitrates, and thrombectomy) during primary PCI in 30 consecutive patients with severe refractory coronary no-reflow (TIMI 0-1, MBG "myocardial blush grading" 0-1). Intracoronary epinephrine administration resulted in significantly improved coronary flow patterns compared to those treated with conventional agents alone. Additionally, the IC epinephrine patients, in comparison to the nonepinephrine group, demonstrated a significant decrease in the 30-day composite of death or heart failure, improvement in ejection fraction, and ST-segment resolution.

Our results suggest the superiority of distal delivery compared to conventional IC delivery, which agrees with Skelding $\boldsymbol{e t} \boldsymbol{a l} .{ }^{(14),}$ who reported that local epinephrine achieved TIMI 3 flow in 69\% of those who developed refractory no-reflow. They reported a significant rise in heart rate but no malignant arrhythmias.

Our findings suggest the efficacy of GpIIb/IIIa inhibitor, which agrees with Prati et al. ${ }^{(15)}$, who evaluated GpIIb/IIIa inhibitor (Abciximab) as a preventive strategy in ACS (acute coronary syndrome) patients for whom an invasive approach was planned. They compared local delivery to in-catheter delivery. The thrombus burden showed a significant reduction in the local delivery arm compared to the catheter arm. In addition, the local delivery group showed significantly shorter postprocedural cTFC, significantly lower 1-year MACE incidence, and had a significantly lower risk of developing procedural MI. Our study uses a combination therapy rather than a single drug regimen to maximize the benefit. 
Our work was almost concordant with Sun et al. ${ }^{13}$ who performed a multivariate analysis on six randomized controlled trials to compare intralesional (IL) and intracoronary (IC) GpIIb/IIIa inhibitor administration in patients with ACS and found that IL administration resulted in improved results for myocardial tissue reperfusion, as demonstrated by an enhanced TIMI flow grade.

In this study, both groups did not show a significant difference regarding MACE during in-hospital stay $(\mathrm{P}=0.07)$ (Table 4), which again agrees with Sun et $\boldsymbol{a l} .{ }^{(13)}$, who found that the IL administration groups had a trend toward MACE reduction, but it was not statistically significant, and that as regard in-hospital major bleeding events, no significant difference among both groups was found.

Diabetes mellitus was found to be the only negative predictor of TIMI flow. This may help with the assessment of patients at risk for no-reflow. Our findings agree with the retrospective analysis of Dharma et al. ${ }^{(16)}$ that was conducted on 856 STEMI patients who had primary PCI and revealed that a higher percentage of patients who had hyperglycemia at the time of presentation showed a final TIMI flow of 0 to 1 .

Similarly, our findings support those of Liu et $\boldsymbol{a l} .^{(17)}$, who assessed no-reflow prognostic factors in 262 individuals with acute STEMI and diabetes after primary PCI. Based on their response to thrombolysis in myocardial infarction (TIMI), patients were classified into two groups: normal flow and no-reflow.

Furthermore, using various statistical methods, related factors were assessed. They found that, compared to the normal flow group, the in-hospital peak glycemia was significantly greater in the no-reflow group. The peak glycemia was also an independent predictor of no-reflow in diabetic patients having STEMI ${ }^{12}$ in a multivariate logistic regression analysis.

\section{LIMITATIONS}

Small-sample size and the study was a single-center study.

\section{CONCLUSION}

Distal coronary artery local injection of a combination of GP IIb/IIIa inhibitors and epinephrine achieved better outcomes in the treatment of no-reflow in comparison with traditional intracoronary administration of the same medications through a guiding catheter. No malignant arrhythmias developed, and thus it was a safe and easy method.

\section{REFERENCES}

1. Hillegass W, Dean N, Liao L (2001): Treatment of no-reflow and impaired flow with the nitric oxide nitroprusside following percutaneous coronary interventions: Initial human clinical experience. J Am Coll Cardiol.,37:1335-1343.
2. Wang H, Lo P, Lin J (2004): Treatment of slow/no-reflow phenomenon with intracoronary nitroprusside injection in primary coronary intervention for acute myocardial infarction. Catheter Cardiovasc Interv.,63:171-176.

3. Fischell T, Maheshwari A (2004): Current applications for nicardipine in invasive and interventional cardiology. The Journal of invasive cardiology, 16:428-32.

4. Assali A, Sdringola S, Ghani M (2000): Intracoronary adenosine administered during percutaneous intervention in acute myocardial infarction and reduction in the incidence of "no-reflow" phenomenon. Catheter Cardiovasc Interv.,51:2731.

5. Gu Y, Kampinga M, Wieringa W (2010): Intracoronary versus intravenous administration of abciximab in patients with ST-segment elevation myocardial infarction undergoing primary percutaneous coronary intervention with thrombus aspiration: the comparison of intracoronary versus intravenous abciximab administration during emergency reperfusion of STsegment elevation myocardial infarction (CICERO) trial. Circulation, 122:2709-2717.

6. Aksu T, Guler T , Colak A(2015): Intracoronary epinephrine in the treatment of refractory no-reflow after primary percutaneous coronary intervention: a retrospective study. BMC Cardiovasc Disord.,15: 10.

7. Eliano P, Navarese T, Frediani L (2021): Efficacy and safety of intracoronary epinephrine versus conventional treatments alone in STEMI patients with refractory coronary no-reflow during primary PCI: The RESTORE observational study. Catheter Cardiovasc Interv., 97: 602- 611.

8. Abu Arab T, Rafik R, El Etriby A (2016): Efficacy and safety of local intracoronary drug delivery in treatment of no-reflow phenomenon: A pilot study. Journal of interventional cardiology,29: 496-504.

9. Gibson C, Schömig A (2004): Angiographic assessment of both epicardial and myocardial perfusion. Circulation, 109:3096-3105.

10. Gibson CM and Cannon CP. (1996): TIMI frame count; A quantitative method of assessing coronary artery flow. Circulation, 93: 879-888.

11. Ito H, Okamura A, Iwakura K (1996): Myocardial perfusion patterns related to thrombolysis in myocardial infarction perfusion grades after coronary angioplasty in patients with acute anterior wall myocardial infarction. Circulation, 93: 1993-1999.

12. Reid J (1986): Alpha-adrenergic receptors and blood pressure control. Am J Cardiol., 28: 6E-12E.

13. Sun B, Liu $Z$, Yin $H$ et al. (2017): Intralesional versus intracoronary administration of glycoprotein IIb/IIIa inhibitors during percutaneous coronary intervention in patients with acute coronary syndromes. A meta-analysis of randomized controlled trials. Medicine (Baltimore), 96: e8223.

14. Skelding K, Goldstein J, Mehta L (2002): Resolution of refractory no-reflow with intracoronary epinephrine. Catheter Cardiovasc Interv.,57: 305-9.

15. Prati F, Di Giorgio A and Manzoli A (2011): Focus on the COCTAIL study. Interv. Cardiol.,3, 275-281.

16. Dharma S, Mahavira A and Haryono N (2019): Association of hyperglycemia and final TIMI flow with one-year mortality of patients with acute ST-segment elevation myocardial infarction undergoing primary PCI. Int J Angiol., 28: 182-187.

17. Liu F, Huang R, Li Y et al. (2021): In-hospital peak glycemia in predicting no-reflow phenomenon in diabetic patients with STEMI treated with primary percutaneous coronary intervention. J Diabetes Res.,66:912- 937 